\title{
An Analysis of Zeno's View Regarding Motion
}

\author{
Dr. Sudipta Chattopadhyay
}

\author{
Govt Approved Lecturer, Malda Women's College, Malda, West Bengal, India
}

\begin{abstract}
Zeno of Elea (about 489 BC), is the favorite disciple of Parmenides (about514 BC). Zeno devotes himself in refuting the views of the opponents of Parmenides. Arguments against the possibility of motion, is first found in 'The Dialectic of Zeno'. To examine the reality of motion, it is necessary to make a new look towards Zeno's reaction on motion. The ancient Greek philosophers are confused to think about space, time and motion. Zeno is the first who deeply thinks about these. His arguments, generally known as his paradoxes, seem to show that motion is simply an illusion which is not possible in any circumstances.
\end{abstract}

Arguments of Zeno are based upon the argument which may be called the antinomy, i.e., a proof that since two contradictory propositions equally follows from a given assumption that must be false of infinite divisibility. But in our times, we know that the fallacies of his proofs, the confusion of the infinite divisibility of space and time with infinite dividedness, he does not notice himself. He argues from the geometrical line to the actual line. Indeed, a geometrical point has no magnitude and a geometrical line is infinitely divisible. But in everyday life the movement between any distance, is not infinitely divisible, nor any observed time which we live through as short or long, is infinitely divisible into moments. Zeno confused an analytic proposition with a synthetic proposition. An analytic proposition does not require any observation to confirm or disconfirm its truth while a synthetic proposition can be true if it corresponds to an actual state of affairs. A distance or time is infinitely divisible, is only an analytic proposition but actual distance traversed in same observed time is not infinitely divisible.

Without motion our usual life would be stopped. Sun, moon, stars, planets and other stars cannot move without motion. If motion is not accepted then astronomy would be in vein. Even we cannot speak and write without the movement of our vocabulary organs and that of fingers respectively. So, the existence of motion has to accept in usual life, in scientific research and in all other aspects of life.

KEYWORDS: Parmenides, Zeno of Elea, Bergson, Kant

\section{INTRODUCTION}

Through his puzzles Zeno proves that the consequences, arising from the assumption of motion, as the Pythagoreans understand it, are more absurd than those arising from the assumption of Parmenides that motion is an irrational delusion of the senses. According to Zeno, true being is to be found, not by sense, but by thought and thought shows that there can be no movement, no change. Therefore, true being is one, excluding of all motion and becoming, as Parmenides says, with no multiplicity in it. Zeno does not directly argue the ideas of Parmenides; he uses tricks with mathematics and infinity to attack plurality and motion. Through his paradoxes, he goes on to prove the impossibility of motion. His puzzles can be seen as part of a comprehensive Elea tic argument against the possibility of motion. Eleatics claim that what is unintelligible cannot exist. According to them, if we suppose that the universe is additive, i.e., made up of parts, then three possibilities may be arise- firstly, the universe may be composed of bodies separated by a void. But this is impossible, because, Parmenides disposes it by arguing that the void is unintelligible and therefore, cannot exist. Secondly, the universe may be composed of indivisible, smallest possible parts (minims) and thirdly, it may be composed of dimensionless points and instants. But Zeno, through his paradoxes, claims that no conceivable assortment of minims and dimensionless points and instants makes possible an intelligible account of motion. Thus, for Eleatics, the universe cannot be additive. If the universe is continuous, i.e., made up, not of parts, but of a continuous, unbroken substance, then, motion can only be explained in terms of compression and rarefaction which are types of change. But for Parmenides change of any kind is impossible, because it involves coming to be, i.e., arising from nothing is void, which is unintelligible and so cannot exist. So, motion is impossible in a continuous universe, too. Therefore, Eleatics claim that no intelligible account of motion can be given; motion cannot occur in any possible universe, no matter what kind of universe we suppose - additive or continuous.

Parmenides writes a poem entitled 'On Nature' which distinguishes between two 'ways' - 'the way of truth' and 'the way of opinion'. Parmenides breaks with the older Ionic tradition by writing in hexameter verse. For Parmenides, his conversion from Pythagoreans to Truth is the central thing in his poem and it is from that point of view we must try to understand his philosophy. According to Parmenides, the most basic and self-evident truth is 'it is'. This is true and one cannot deny it without falling into logical contradiction. What he means by 'it is' is that 'what is, is' which is known as the 'principle of identity', i.e., something is what it is or A is A. He makes Being or existence into a predicate, so that 'it is' means Being is real. 'What is' is and therefore, cannot be anything other than what it is. On the other hand, 'what is not' cannot be existent. For Parmenides, reality is an absolute and unchanging whole and motion, space and plurality are simply illusions. He thinks that the absolutely real is Being, Not-being is the unreal. Not-being is not at all and it is identical with becoming, with the world of shifting and changing things. The world is revealed to us by our senses. The essentials of the world of sense are multiplicity and change. So, the world is unreal, illusory, a mere appearance and therefore not-being. Only Being truly is and it is the Being which is the first principle of things excluding 
of all becoming. True Being is absolutely one, there is no multiplicity in it. It is absolutely static and unchangeable; there is no motion in it. For him, change means that something has passed from being one thing to another and therefore is not that original thing; it will soon change into something else. This constant change that we see every day does not actually happen and cannot be considered real. He claims that the universe is one which is completely unchanging with one beginning or end and there is nothing like time or motion. He thinks the universe as a sphere, completely solid, with no room for motion or ability for change. But his ideas on change are completely wrong. Change does not necessarily mean something no longer exists. Since matter is never created or destroyed then the way we get new things is through change. Our world is in a constant state of change. Therefore, the ideas that everything we see is an illusion and that the universe is one and unchanging are false.

Heraclitus claims reality as change, flux and becoming. His philosophy is fundamentally different from that of Parmenides. He admits logos as the basic order of things. Parmenides believes in the oneness of the universe which is somewhat of a similarity and his point of view is much more scientific than Heraclitus. Parmenides uses logic and reason to draw conclusions from truths. Though the conclusions that the Elea tic philosophers reaches are wrong, it is their methods that has a lasting influence. They are trying to use reason to determine truth. Instead, the one-sided doctrine of Parmenides is unacceptable, as also is the one-sided doctrine of the Pythagoreans. To get the knowledge of the universe, both factors, i.e., the One and the Many, Stability and Change, are valuable. Though, Heraclitus is trying to solve in a philosophy that claims to do justice to both elements, one and many, through a doctrine of Unity in Diversity, Identity in Difference, his philosophy is also unsatisfactory. We have to give importance to the stable element in things, as well as to the flux or change of things, otherwise, a confliction between the materialistic monism with the immaterial highest being, may arise.

Zeno defends the doctrine of Parmenides in an indirect way, by composing a prose treatise. His refutation of the general conception of the world is so acute that Aristotle calls him the inventor of dialectic. His proofs are directed partly against the assumption of a multiplicity of things and partly against motion.

\section{DISCUSSION:}

Now, if we concentrate on Zeno's paradoxes, we can see that among his eight surviving paradoxes which are presented in Aristotle's 'Physics', many are equivalent to one another but four of them are the most famous which are as follows:

1. In order to travel a distance, if an object wants to go to the other side, it has to travel an infinite number of points, in finite times. But it is not possible to travel an infinite number of points and so an infinite distance, in a finite time. Therefore, no object can travel any distance, whatsoever and motion is impossible.

This argument of dichotomy claims the impossibility of motion by asserting that any object which is in locomotion, must arrive at the half way stage before it arrives at the goal. The object is required to travel an infinite series of distances, which is impossible. In order to travel each of the distances involved, the object requires an infinite time, i.e., an infinite duration and so it can never be completed. We find the 'dichotomy' as being intrinsically linked with the conception of the infinite divisibility of space as well as of time. Here, time is assumed as consisting of infinitively small, 'atomic' moments. During each of the atomic moments, movement cannot take place as infinitively many instances of space will have to be transgressed. The moved object also does not change during the course of motion. In this regard, what is true for one 'time-instant' is also true for each preceding and each succeeding one. Motion can never have started until there is a change in the conditions of time and space as they have been assumed. If time and space both are held to be continued, the impossible conclusions of Zeno's arguments will not follow.

2. If an Achilles and a tortoise run a race and the tortoise is given a start, Achilles, despite his greater speed, cannot reach the tortoise, for while Achilles is moving from his initial starting point to the tortoise's initial starting point, the tortoise has moved a certain distance beyond and the same holds for all subsequent intervals. This argument shows the impossibility of passing a moving goal.

The tortoise must travel at least one point of space in each moment, i.e., an infinite number of points, in an infinite series of moments, i.e., in infinite time. In order to overtake the tortoise, Achilles must travel each spatial point through which the tortoise has passed, i.e. an infinite distance. In modern times, space and time are not regarded as separate entities. In science, scientists talk of events or space-time continūm, while Zeno thinks space and time as separate entities. For him, infinite parts of space to be traversed will require infinite moments of time, which is not correct.

3. The paradox of the flying arrow points out a contradiction. An object cannot be in two places at the same time. Therefore, at any particular moment in its flight the arrow is in one place and not in two. But to be in one place, is to be at rest. So, in each and every moment of its flight the flying arrow is at rest. It is at rest throughout the flight and therefore, motion is impossible.

This argument claims that a moving arrow can neither move in the place where it is not, nor in the place where it is. Anything is at rest when it is at a place equal to itself. The arrow is always at a place equal to itself; therefore, the arrow is always at rest and can never move. In this case, it is difficult to say whether a moving thing remains same during the different instants of time when it occupies different places, or not. If a thing remains same, then it is difficult for us to assert the identity of the different things occupying separate positions of space, of what we denote as 'a moving thing'; rather, these are different things at different places, which we arbitrarily connect. "The arrow remains the same during the motion' indicates that it remains same with all its attributes with the part of space which it occupies, for if an attribute is changed, we cannot speak of the 'same' thing. Therefore, if we assert that the moving arrow remains really the same during the movement, we feel difficulties to explain how it could ever move at all. Zeno would have balked at the idea of motion at an instant and Aristotle explicitly denies it believing that motion occurs only over duration of time and that duration divides into intervals but never into indivisible instants. However, in calculus, the derivative of position $\mathrm{x}$ 
with respect to time $t$, namely $\mathrm{dx} / \mathrm{dt}$, is the arrow's speed, and it has non-zero values at specific places at specific instants during the flight. The speed during an instant would be $0 / 0$ and so is undefined. Using these modern concepts, Zeno cannot successfully argue that the speed of the arrow is zero at every instant. Thus, we find that Zeno's Arrow Paradox has false assumptions and so is unsound.

4. At the same time, two lines, each composed of an equal number of bodies of equal size, can travel same distance, with same velocity. Among the two lines, if one is at rest and another travels by the first one, then the unit of time will be the time of travelling the length of that line. But if a third line travels from the opposite side of the second line, then each of the two lines, will travel the length of the static line in half of the main unit.

If two lines $P$ and $Q$ travel same distance with same velocity, $P$ is at rest and $Q$ travels by $P$, then the unit of time(for $Q$ ) will be the time of travelling the length of $P$. But if a third line $R$ (composed of an equal number of bodies of equal size to $P$ and Q) travels from the opposite side of $Q$, then each of $Q$ and $\mathrm{R}$ will travel the length of $\mathrm{P}$, in half of the main unite. Thus, Zeno proves that the half of a certain time is equal to the whole (i.e. double) of that time. But this type of conclusion is not possible. We have to remember that the interpretation 'half of a certain time is equal to the whole of that time' is not of Zeno but of Aristotle who misunderstands this paradox. About this paradox Sir David Ross has said that "Very difficult to follow, partly owing to use of ambiguous language by Aristotle, partly owing to doubts as to the readings."1

Modern exponents claim that Zeno does not say that Q will pass $\mathrm{P}$ (stationary) and $\mathrm{R}$ (moving at the same speed as $\mathrm{Q}$ but in the opposite direction) in the same amount of time, rather he says that if $Q$ is travelling at, e.g., a speed of one point of space per unit of time, it will pass one point of $\mathrm{P}$ in one unit of time, but it will pass one point of $\mathrm{R}$ in half a unit of time; thus dividing the indivisible unit which is impossible. Here, the issue of relative velocity is irrelevant.

Space is thought to be either discrete or continuous in Greek Philosophy. Now, if space is discrete then there should be smaller units of it and if it is continuous then it is infinitely divisible. Zeno disproves the ideas of discrete and continuous space through his paradoxes. His 'Arrow paradox' disproves how space can be discrete. The arrow is either in motion or at rest in one smaller unit of space. It can move, because for motion to occur, it would have to be in one smaller unit of space at the start of an instant and at another at the end of the instant. However, this means that the instant is divisible, which is impossible because by definition, instants are indivisible. Hence, the arrow is always at rest in one of the smaller unit of space. Space therefore, cannot be thought of as discrete, otherwise it must be continuous. 'The paradox of Achilles and the tortoise' disproves how space can be continuous. In a race, if Achilles is at the starting point of the race and a tortoise is at the halfway point, then though Achilles can run faster than the tortoise, he will never catch up to win the race; by the time Achilles reaches the halfway point, the tortoise will be in another point and this will go again and again. Therefore, Achilles will never win the race. Space is therefore, not continuous and philosophers are left pondering upon the correct notion of it. Zeno thus shows how to take reasonable assumptions and create absurd conclusions. This is called
Zeno's dialectic. He is the first to bring upon this type of reduction to absurdity which is used in all types of logic and philosophical research today.

The Pythagorean Science is basically atomistic which claims that the universe is additive, i.e., composed of atoms or minims, indivisible smallest-possible units of space and time. Pythagoreans maintain both, that the world is composed of atoms and that any magnitude is infinitely divisible. But there is a contradiction, if it is taken to have definite magnitude, then, there will be lines which cannot be bisected and no magnitude will be infinitely divisible. On the other hand, if the atoms are made dimensionless to give infinite divisibility, no quantity of such atoms can ever add up to any magnitude at all. The negation of their view of space and time, i.e., of all dividedness in space and successiveness in time, might become the fundamental thought of Zeno. Robert Brumbaugh, in 'The Philosophers of Greece', claims that "Zeno's paradoxes were designed to bring out the inherent absurdities of such a world view and to show that, however one interpreted this position, whichever of its premises one adopted, no account of motion could be given which did not end in absurdity, whether space and time were atomistic or infinitely divisible, no intelligible account of motion through them was possible." 2

Zeno, as a disciple of Parmenides, believes that motion is an illusion and is impossible. Beside this, through his arguments, his aim is to prove that even on the pluralistic hypothesis, motion is equally impossible. He says that "The Real is a plenum, a complete continuum and motion is impossible. Our adversaries assert motion and try to explain it by an appeal to a pluralistic hypothesis. I propose to show that this hypothesis does nothing to explain motion, but only lands one in absurdities." 3

Zeno's arguments against multiplicity and motion are based in the principle of contradiction and the presupposition that the same thing must not be affirmed and also denied. Though this principle and presupposition is not abstractly expressed but applied with clearness and certainty. His arguments are so logically well arranged, having a crucial role in the developed technique of refutation that the Eleatic school gets an evidence to attain the technique. Indeed, by saying that motion and multiplicity are not real, Zeno wants to say that certainly there is motion and multiplicity but they are not true. Accordingly, the world is here, is present to our senses, but it is not the true world. It is not reality but mere appearance or illusion which hides the real being of things. For him, the objects around us have existence but not selfexistence, not self-substantiality; they are rather beings whose being flows into them from another, i.e., from reality which itself is self-existent and self-substantial. Motion and multiplicity are mere appearances of the reality. Though Zeno does not speak of appearance and reality in this way but this is what he is looking for. His philosophy is the completion of the Eleatic principle, as well as, the beginning of its end. He takes up the antithesis of being and non-being so acutely that the inner contradiction of the principle becomes much more prominent with him than even with Parmenides.

F. H. Bradley shows that the categories of human thought are puzzled with contradiction. Likewise, Nāgārjuna claims that the categories of space, time and substance are shot through with contradiction. 
The arguments of Zeno against motion succeed to create a deep impression on our mind. Zeno wants to support the view of Parmenides, but his method to achieve this goal develops his dialectic and gives importance to the discussion of the problems regarding the ideas of space, time and motion. Zeno's all arguments are based upon the one argument which may be called the antinomy (i.e., a proof that since two contradictory propositions equally follow from a given assumption that must be false) of infinite divisibility. But in our times, we know that the fallacies of his proofs, the confusion of the infinite divisibility of space and time with infinite dividedness, he does not notice himself. He argues from the geometrical line to the actual line. Indeed, a geometrical point has no magnitude and a geometrical line is infinitely divisible. But in everyday life the movement between any distance, is not infinitely divisible, nor any observed time which we live through as short or long, is infinitely divisible into moments. Hence Zeno's paradox is due to the confusion of two standpoints. In other words, we can say that Zeno confused an analytic proposition with a synthetic proposition. An analytic proposition does not require any observation to confirm or disconfirm its truth while a synthetic proposition can be true if it corresponds to an actual state of affairs. A distance or time is infinitely divisible, is only an analytic proposition but actual distance traversed in same observed time is not infinitely divisible.

Henri Bergson revives the metaphysics of Heraclitus by accepting the ever-changing vital process as the supreme reality. Zeno and Bergson's theories to the study of time have inter-related the perspective calculus and added to the knowledge base of abstract thought. Calculus, the theory based on experimental data and analysis, describes motion or any rate of change. Mathematicians claim that calculus is not abstract mathematics but is exact the prediction which may be of falling bodies or particles' motion. Indeed, we can understand the nature of time, but when we speak, words fail to describe the abstract image of time. Beside this, we can see that philosophers and scientists observe many common attributes of time but draw different conclusions. Thus, time is a paradox by the various valid theories and images made, though none of them can be fully accepted.

The most important paradox in mathematics is the motion of an object at an instant. Our common sense binds us to think that the speed of an object at an instant is zero, as there is no change in time. In order to find the derivative, the instantaneous rate of change of an object's position, one must have a continuous function, i.e., continuous time and space. Zeno's paradox of the arrow states that if everything is either at rest or at motion, when it occupies a space equal to itself, every instant of its flight the moving arrow is at rest. Thus, Zeno's thought experiment of the arrow excludes the idea of speed of an object at an instant. In terms of calculus, an instant is a point. A point has no dimensionality but there is unlimited number of points in the arrow's flight. Bergson, in terms of cognitive and subconscious processes, advances the philosophical response to non-motion within motion. He says that "We express duration in terms of extensity, and succession thus takes the form of a continuous line or chain, the parts of which touch without penetrating one another."4

According to Bergson, duration is a continuous sequence, just as calculus requires a continuous function in order to derive the function. He argues that space alone is homogeneous, pure duration can only be experienced in the mind through intuition; the inability to identify the rate of change in the present instant is the evidence of the limiting ability of our perception. He realizes the importance of the paradox of motion at an instant, his concept of pure duration gives philosophical content to the abstract world of calculus. Indeed, Zeno and Bergson approach the problem of the derivative in different ways but they give support to the principles of calculus to better enrich the abstract image of time. Bergson, from his metaphysical standpoint, criticizes the notion of specialized time. For him, real time is duration in which each moment of time melts into another without a break. So, it might be said that Zeno confuses real duration with the unreal specialized time. Again, he confuses the legitimate distinction of space and time in thought with inseparable space-time continum. According to linguistic convention, movement is always related with parts of a distance and moments of time, movement cannot be reduced to separate parts of space or moments of time. Space and time can be distinguished in thought, but cannot be separated in reality. Now, it is important to see that whether Zeno's theory of motion has any similarities with that of other great thinkers or it is it-self contradictory. David Hume does not admit the infinite divisibility of space and time by claiming that they are composed of indivisible units having magnitude. But it is impossible to conceive of units having magnitude which are yet indivisible and it is not satisfactorily explained by Hume. Kant argues that the contradictions that Zeno shows are immanent in our conceptions of space and time. Space and time are forms of our perception, they are not real; they do not belong to things as they are in themselves, but rather to our way of looking at things. Our minds impose space and time upon objects, but objects do not impose space and time upon our minds. Kant claims that comprehending the infinite is beyond the capacity of human reason, when we try to think the infinite, whether the infinitely large or the infinitely small, we fall into contradictions. His first publication, 'Thoughts on the True Estimation of Living Forces' (1746), attempts to solve controversy that has been contested ever since Leibniz's attack on Descartes' laws of motion in the 'Acta Eruditorum' (1686). Kant argues for changes of bodily states by maintaining that mutual changes of state require mutual interaction. Through his 'Physical Monadology' (1756), he reconciles the infinite divisibility of space. The first chapter of his 'Metaphysical Foundations of Natural Science' (1786), the 'Phoronomy', considers the quantity of motion of matter and how it is to be constructed in a-priori intuition.

Kant claims that due to the relativity of space "The composition of two motions of one and the same point can only be thought in such a way that one of them is represented in absolute space and instead of the other, a motion of the relative space with the same speed occurring in the opposite direction is represented as the same as the latter."5 It considers three possible cases for the composition of two motions, they are: (i) the two motions are in the same direction, (ii) the two motions are in opposite directions and (iii) the two motions enclose an angle. Kant tries to show how one can construct a-priori intuition in a single motion out of the two motions, described in the three cases mentioned above. The third chapter, the 'Mechanics', concerns how it is possible to experience matter as having a moving force, i.e., how one matter communicates its motion to another by means of its moving force. Kant states his First Law of Mechanics as "The total quantity of matter remains 
the same throughout all changes in matter."6 Kant's Second Law of Mechanics is "Everybody persists in its state of rest or motion, in the same direction, and with the same speed, it is not compelled by an external cause to leave this state."7 It is much closer to Newton's 'Law of Inertia' but not identical with it. I have discussed earlier that the 'Law of Inertia' asserts that an object at rest will remain at rest unless acted upon by an external and unbalanced force, as well as, an object in motion will remain in motion unless acted upon by an external and unbalanced force. Kant's Third Law of Mechanics asserts the equality of action and reaction in the communication of motion. Through the final chapter, the 'Phenomenology', Kant focuses on how the motion of matter can be experienced modally. Hegel compares Zeno with Kant by saying that "Kant's antinomies do no more than Zeno did here. "8 Hegel believes that, to be satisfactory, it is necessary for a solution to make room for both sides of the contradiction. A satisfied solution is only possible by rising above the level of the two opposite principles and taking them both up to the level of a higher conception, in which both opposites are reconciled. He regards Zeno's paradoxes as examples of the essential contradictory character of reason. For him, all thought and reason contain immanent contradictions that they first posit and then reconcile in a higher unity. The contradiction of infinite divisibility is reconciled in the higher notion of quantity which contains two factors, i.e., one and many. It is clear that Hume, Kant and Hegel are trying to give solution to solve Zeno's paradoxes. Their solutions have been very stimulating to subsequent thinkers but ultimately have not been accepted. In modern times, mathematicians, physicists and philosophers are eager to know what steps are necessary in order to escape the contradictions shown by Zeno's fruitful paradoxes. The concepts of space, time and motion have to be radically changed and the mathematical concepts of line, number, measure and sum of a series have to be give a new look. Indeed, Zeno's integers have to be replaced by the contemporary notion of real numbers. The new onedimensional continum, the standard model of the real numbers under their natural order, is a radically different line than what Zeno imagines. The new line is the basis for the scientist's notion of distance in space and duration through time and it is no longer a sum of points, as Zeno supposes, but a set-theoretic union of a non-denumerable infinite number of unit sets of points. This is the way through which we can make a sense of higher dimensional objects like the one-dimensional line and the two-dimensional plane, being composed of zero-dimensional points. Zeno argues that a simple sum of an infinity of zeros would never total more than zero. But the density of points in a line is so high that no point is next to any other point and the infinity of points in the line is much more than any infinity Zeno could have imagined. Now, the sum of an infinite series of numbers can have a finite sum, unlike in Zeno's day. Moreover, the fallacies of Zeno paves the way for new philosophical thought and are proved very fruitful in farther thinking about motion; there emerge in us, a capacity to think about the reality of the concept of motion.

\section{CONCLUSION:}

To be in motion, an object has to move from one place to another place, with reference to time. In the language of physics also, we can say that motion is a change in position of an object over time. In mathematics, motion is described in terms of displacements, distance, time, velocity, speed and acceleration. We cannot deny the existence of motion. Our general sense asserts that any object in this universe stands with reference to space-time continūm. To be in motion, an object has to move from one place to another place, with reference to time. It indicates the change of its position, with reference to time.

In our times, we know from science that Earth, Sun and other planets and stars move on their own orbits. If motion is not accepted, then, it would not be possible and astronomy would become false. I have discussed earlier that NASA's Kepler mission has confirmed the first near-Earth-size planet, Kepler-452b in the habitable zone around a Sun-like, G2-type star. This discovery and the introduction of 11 other new small habitable zone candidate planets mark another milestone in the journey to finding another Earth. Kepler$452 \mathrm{~b}$ is larger than Earth, its distance from its parent star is 5 percent farther than the distance between the Earth and the Sun. Kepler-452 is 6 billion years old, 1.5 billion years older than our sun. It has the same temperature, and is 20 percent brighter and has a diameter 10 percent larger. John Jenkins' Kepler data analysis leads at NASA's Ames Research Center in Moffett Field, California. For him, we can think of Kepler-452b as an older, bigger cousin to Earth. Kepler- $452 \mathrm{~b}$ is the smallest planet to date discovered orbiting in the habitable zone, the area around a star where liquid water could pool on the surface of an orbiting planet, of a G2-type star, like our Sun. Including this planet, the total number of confirmed planets become one thousand and thirty.

In practical life, human beings and other animals change places for various purposes, e.g., food, shelter, learning, etc. If there is no motion, how would it be possible for them to lead a normal life as without movement they cannot change place. If there is no motion, then, we cannot speak with others. To talk to others the movement of our vocabulary organs, i.e., lips and tongue, has to accept and the movement indicates motion. Again to write, the movement of fingers also indicates motion. Hence, this discussion proves that motion has reality and it exists.

\section{REFERENCES:}

[1] 'A History of Philosophy': Vol. I: Part 1: Frederick Copleston, S.J.: Image Books: Garden City, New York: 1962: p.74.

[2] 'Zeno and Naagaarjuna on motion': Philosophy East and West 26, no.3: Mark Siderits and J.Dervin O'Brien: The University Press for Hawaii: 1976: p.282.

[3] 'A History of Philosophy, Vol. I': Frederick Copleston: Garden City, New York: Paperback ed.2003: p.58.

[4] 'Time and Free Will: An Essay on the Immediate Data of Consciousness: Vol .1': Henri Bergson: F. L. Pogson (Trans.): George Allen and Unwin Ltd., London: Humanities Press Inc., New York: $1^{\text {st }}$ ed.1910, $3^{\text {rd }}$ ed. 1913: p.101.

[5] 'Metaphysical Foundations of Natural Science': Immanuel Kant: Michael Friedman (ed.): Cambridge University Press: Cambridge: $1^{\text {st }}$ ed.2004: p.26.

[6] Ibid: p.80.

[7] Ibid: p.82.

[8] 'Conspectus of Hegel's Book: Lectures on the History of Philosophy': Volume XIII: Volume I of The History of Philosophy: History of Greek Philosophy: Vladimir Ilyich Lenin: Stewart Smith (ed.): Progress Publishers: Moscow: $4^{\text {th }}$ ed.1976: p.326. 